\title{
A Palmprint Recognition Method Based on Gabor Wavelet and Local Direction Pattern
}

\author{
Mianshui $\mathrm{Yu}^{1, \mathrm{a}}$, Xiaomeng $\mathrm{Xie}^{2, \mathrm{~b}}$ \\ ${ }^{1}$ Guangdong College of Industry \& Commerce, Guangzhou 510510, China \\ ${ }^{2}$ College of Computer, South China University of Technology 510641, China \\ acmsyu@163.com, bxmchf@126.com
}

Keywords: Palmprint Recognition, Local Direction Pattern, Gabor wavelet

\begin{abstract}
Palmprint recognition is an emerging biometric technology that identifies a person based on the hand image. In this paper, a novel palmprint identification method based on Gabor wavelets and Local Direction Pattern (LDP) techniques has been proposed. The Gabor response images at different scales and directions are obtained by convolving the multi-channel Gabor wavelet and palmprint images, which are further divided into non-overlapping sub-blocks. The LDP characteristics are extracted from each sub-block and the feature vector of palmprint image is then produced by combining them using the LDP operator. Finally, the palmprint images are identified using the nearest neighbor classification method. The proposed method has been verified on the public PolyU palmprint database, and the experimental results have shown that the proposed method is effective.
\end{abstract}

\section{Introduction}

Palmprint recognition is a relatively new biometric identification technology that has been applied to person identification in recent years. Compared to other biometrics used in person identification, the palmprint has many unique advantages, such as, harmlessness, concealment and convenience. A highly accurate human identification can be achieved by analyzing the human palm, which is useful in meeting the requirements of high security level authentication. Therefore, palmprint recognition is a biometric method with broad application prospects and it is widely studied nowadays [1].

Typically, a palmprint recognition system involves palmprint acquisition, preprocessing, feature extraction, and matching. The feature extraction is the core and the most complex part of the recognition system. Based on different feature extraction and matching methods, palmprint recognition methods can be roughly classified into structure-based methods, statistics-based methods, subspace-based methods, and texture/transform domain feature-based methods[2][3]. Shu et al. [4][5] used 12 line detectors to detect the palm ridges around all directions, represented by a linear fitting technique; and then extracted the palmprint features such as endpoints, the intercepts and the tilt angles of the line segments; finally, the resulting features were classified based on Euclidean distance. Most of the structure-based methods involve fingerprint identification that is simple and intuitive; however, the performance of these methods is not ideal because of the limiting factors like translation, rotation and sensitivity to noise. Pand et al. [6] and Li et al. [7] used translation invariant Zernike moments to extract palmprint features. This type of methods has the characteristic of translation or rotation invariance in palmprint image to effectively avoid the image translation and rotation due to the preprocessing. The methods based on statistics are easy to model the feature extraction procedure and these are less sensitive to noise. However, such methods do not contain structural information, hence lose a lot of discrimination information, which leads to unsatisfactory performance. Wu et al. [8] proposed the "FisherPalm" method by applying dimension reduction of Linear Discriminant Analysis (LDA) based on the Principal Component Analysis (PCA). Du et al. [9] used the horizontal and vertical Two-Dimensional LDA (2DLDA) to extract the Gabor features of the palmprint images, and used distance-based adaptive strategy to combine these two features. The resulting high recognition rate confirms the validity of subspace-based methods; however, this method usually 
requires many training samples for each category and the selection of the training samples has significant impact on the recognition results. Palmprint images have a lot of texture information, including the principal lines, wrinkles, delta points and other minutiae, and can be regarded as a texture images. Kong et al. [10] used a method called "Competitive code" that filters palmprint images with real-valued Gabor filter in six directions, and encodes the direction that has the smallest magnitude. Mu et al. [11] used Local Binary Pattern (LBP) to extract the palmprint features and Fisher Linear Discriminant (FLD) classifier to learn and label the features. These methods have a high recognition rate that indicates the efficacy of the texture based methods.

LBP is a simple and effective local feature representation method that has been successfully applied in face recognition and texture analysis. However, LBP is sensitive to non-monotonic illumination changes and random noise, which ultimately affects the recognition performance. Shojaiee et al. [12] proposed a method named Local Composition Derivative Pattern (LCDP) that gets the direction information missed in the LBP by extracting the first derivative in the radial and other directions in an image. Based on LBP method, Jabid et al. [13] proposed a new image texture characterization method called Local Direction Pattern (LDP), which has been successfully used in face recognition. Combining the advantages of Gabor wavelet and LDP, this paper presents a novel palmprint recognition method, named Gabor Local Direction Pattern (G-LDP). The Gabor wavelet is used to filter the palmprint images and obtain Gabor wavelet decomposition images in different directions and scales; then the histogram features are extracted from these images using LDP operators. To obtain more local details, each image is divided into non-overlapping blocks, and the LDP features are extracted from each block. Afterwards, all of the features from sub-blocks are combined together to form the final feature vector. The proposed method is verified on the PolyU database of Hong Kong Polytechnic University, and the validity of the method has been confirmed from the results.

\section{The Proposed Method}

Gabor wavelet. The Gabor filter was first proposed by Dennis Gabor in 1946. The Gabor wavelet kernel function has similar characteristics as the reflection area of the human cerebral cortex. For instance, it can obtain the local information of spatial frequency, spatial location, and the orientation. Therefore, it has been widely used in the field of computer vision and image analysis [14] [15]. 2D-Gabor wavelet can be expressed as [16]

$$
\psi_{\mu, \nu}(\zeta)=\frac{\left\|k_{\mu, \nu}\right\|^{2}}{\sigma^{2}} e^{\left(-\left\|k_{\mu, \nu}\right\|^{2}\|\zeta\|^{2} / 2 \sigma^{2}\right)}\left[e^{i k_{\mu, \nu} \zeta}-e^{-\sigma^{2} / 2}\right]
$$

Where, $\zeta=(x, y)$ represents the position of each pixel in the input image, $k_{\mu, v}=k_{v} e^{i \varphi_{\mu}}, k_{v}=k_{\max } / f^{v}$ represent the corresponding frequency, $\varphi_{\mu}=\mu \pi / 8 \in[0, \pi)$ indicates the direction, and $i$ is the imaginary unit $\left(i^{2}=-1\right)$. Therefore, $k_{v}$ and $\varphi_{\mu}$ indicate the scale and direction of the filter, respectively.

Gabor feature of palmprint image is obtained by the convolution of palmprint image and the Gabor filter. In the proposed method four frequencies $(v \in 0, \cdots .3)$ and six directions $(\mu \in 0 . \cdots .5)$ are used to obtain 24 Gabor filters. Suppose $\mathrm{f}(\mathrm{x}, \mathrm{y})$ is a palmprint image, its convolution with a Gabor filter is expressed as

$$
G_{\psi f(x, y, \mu, v)}=f(x, y) * \psi_{\mu, v}(\zeta)
$$

Where, (-*-) denotes the convolution operation. By applying the convolution for all 24 filters, and then considering the amplitudes as Gabor characteristics, a 24-dimensional wavelet feature vector is obtained at each point in the image. 
Local Directional Pattern. LDP operator is a texture characterization method proposed by Jabid et al. [13] based on the Local Binary Pattern (LBP). For a $3 \times 3$ rectangular block, the LDP value at its center is calculated as: Firstly, the edge response values in eight directions ( $\mathrm{m} 0 \ldots \mathrm{m} 7)$ are obtained using the Kirsch operators in eight directions (M0 ... M7, as shown in Fig. 1); Secondly, the first $k$ responses that have the maximum absolute values can be selected and set to 1 , while the other (8-k) responses are set to 0, producing the important edge response information; Finally, the clockwise or counterclockwise encoding information of these eight binary values are considered as the $\mathrm{LDP}_{\mathrm{k}}$ value at the center of the $3 \times 3$ rectangular block . Based on the above procedure, the $\mathrm{LDP}_{\mathrm{k}}$ can be defined as:

$$
L D P_{k}=\sum_{i=0}^{7} b_{i}\left(m_{i}-m_{k}\right) \cdot 2^{i}, b_{i}(\alpha)=\left\{\begin{array}{l}
1, \alpha \geq 0 \\
0, \alpha<0
\end{array}\right.
$$

Where, $m_{k}$ is the larger response value of the $k^{\text {th }}$ direction. LDP operator will generate $C_{8}^{k}$ different encoding values, i.e., in the 8 bit binary code, only $k$ bits are 1 and the remaining are 0 . Fig. 2 shows an example of calculating the LDP with $k=3$.

$$
\begin{aligned}
& {\left[\begin{array}{lll}
-3 & -3 & 5 \\
-3 & 0 & 5 \\
-3 & -3 & 5
\end{array}\right] \quad\left[\begin{array}{rrr}
-3 & 5 & 5 \\
-3 & 0 & 5 \\
-3 & -3 & -3
\end{array}\right]\left[\begin{array}{rrr}
5 & 5 & 5 \\
-3 & 0 & -3 \\
-3 & -3 & -3
\end{array}\right] \quad\left[\begin{array}{rrr}
5 & 5 & -3 \\
5 & 0 & -3 \\
-3 & -3 & -3
\end{array}\right]} \\
& \text { East } M_{0} \text { NorthEast } M_{1} \text { North } M_{2} \quad \text { NorthWest } M_{3} \\
& {\left[\begin{array}{rrr}
5 & -3 & -3 \\
5 & 0 & -3 \\
5 & -3 & -3
\end{array}\right] \quad\left[\begin{array}{rrr}
-3 & -3 & -3 \\
5 & 0 & -3 \\
5 & 5 & -3
\end{array}\right]\left[\begin{array}{rrr}
-3 & -3 & -3 \\
-3 & 0 & -3 \\
5 & 5 & 5
\end{array}\right] \quad\left[\begin{array}{ccc}
-3 & -3 & -3 \\
-3 & 0 & 5 \\
-3 & 5 & 5
\end{array}\right]} \\
& \text { West } M_{4} \text { SouthWest } M_{5} \text { South } M_{6} \text { SouthEast } M_{7}
\end{aligned}
$$

Fig.1 Kirsch operators of eight directions

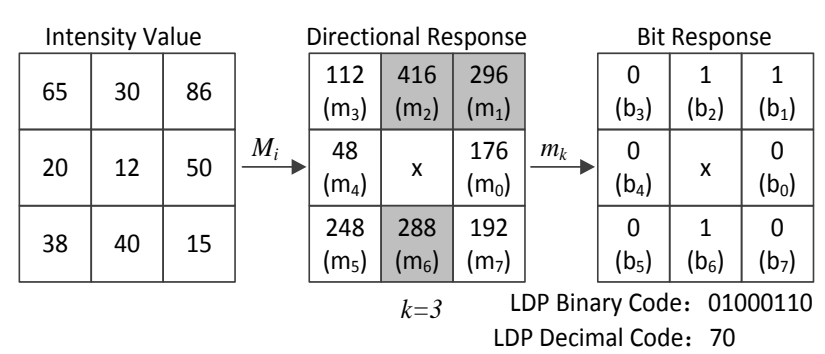

Fig.2 Calculation of LDP value $(k=3)$

Palmprint Feature Extractions. The overall framework of palmprint feature extraction is shown in Fig.3. The preprocessed palmprint image is convoluted with Gabor filter, generating 24 Gabor feature images with different scales and directions. Then LDP operator is used to encode each image to obtain the LDP-encoded images. The LDP feature is usually expressed by a histogram H (i) as given in (4):

$$
H(i)=\sum_{x=1}^{M} \sum_{y=1}^{N} f\left(L D P_{k}(x, y), i\right), f(\alpha, i)=\left\{\begin{array}{l}
1, \alpha=i \\
0, \alpha \neq i
\end{array}\right.
$$

Where, $\operatorname{LDP}_{k}(x, y)$ is the LDP value of the pixel $(x, y)$. In this paper, $k=3$ is chosen that correspond to detection of three main edge responses; therefore, the histogram of each encoded image will contain $56\left(C_{8}^{3}=56\right.$ ) bins. Although the LDP histogram contains rich texture information of the image, the LDP histogram of the entire image does not have location and space information. Thus, the image is divided into $n$ non-overlapping blocks: $R_{0}, R_{1} \cdots R_{n-1}$, and the histogram $H_{R_{i}}$ is calculated for each block, respectively. The resulting $n$ histograms are combined to form the feature vector of each Gabor image. Finally, these LDP feature vectors of the Gabor feature images are combined to form a G-LDP palmprint image feature vector. 


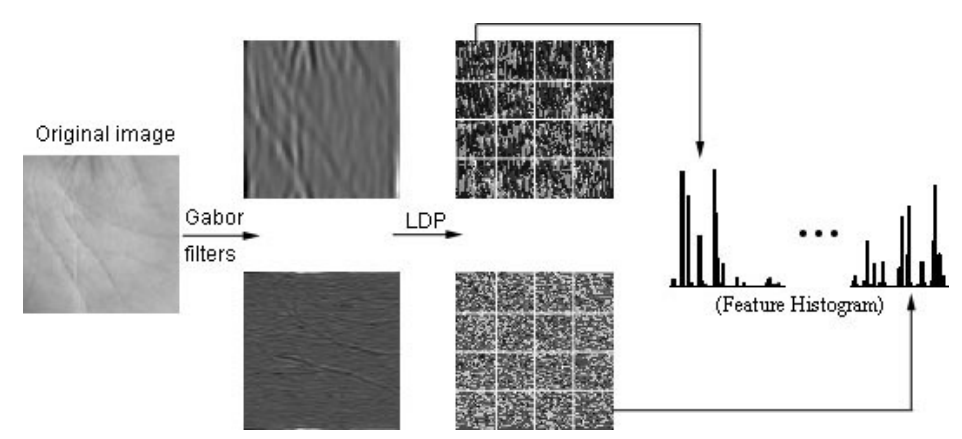

Fig. 3 Overall framework of palmprint feature extraction

To compare the G-LDP feature vectors of two palmprint images, the Chi-square test is applied to calculate the similarity between them, and then the nearest neighbor classifier is used to classify the images. The Chi-square test is defined as follows.

$$
\chi\left(H_{1}, H_{2}\right)=\sum_{i} \frac{\left(H_{1}(i)-H_{2}(i)\right)^{2}}{H_{1}(i)+H_{2}(i)}
$$

\section{Experimental Results and Analysis}

The PolyU palmprint database [17] from the Hong Kong Polytechnic University is used to verify and evaluate the performance of the proposed method. The database contains 7752 palmprint images collected from 386 palms of 193 subjects. The palmprint images of each person were collected twice with a time interval of two months. The Region Of Interest (ROI) with a size of $128 \times 128$, is extracted after preprocessing the palmprint images. Fig. 4 shows an example of ROI of some of the palmprints in the database. In the experiment, 2000 palmprint images for 200 subjects were randomly selected as samples from the database, where 10 images for each palm were used for the experiment. Experiments were carried out in MATLAB software environment.
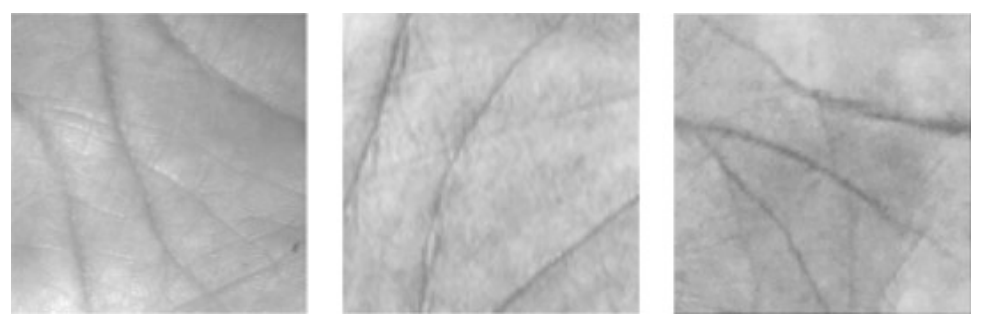

Fig. 4 Illustration of palmprint ROI image in the database

Three different block sizes $(4 \times 4,8 \times 8$, and $16 \times 16)$ were used to evaluate the effects of block size on the performance of LDP operators. Table 1 shows the results with different block sizes. It can be observed from the Table 1, that the recognition performance improves with the increasing size of the blocks; however, the length of the feature vectors also significantly increases with the block size, which also affects the performance of the algorithm. Among the three sizes, the $8 \times 8$ is found to be a relatively appropriate choice.

Table 1 Result of LDP with different block sizes

\begin{tabular}{c|c|c}
\hline Block size & $\begin{array}{c}\text { Feature } \\
\text { vector length }\end{array}$ & Average recognition rate \\
\hline $4 \times 4$ & 896 & 95.75 \\
$8 \times 8$ & 3584 & 98.65 \\
$16 \times 16$ & 14336 & 96.35 \\
\hline
\end{tabular}


In order to further verify the effectiveness the proposed algorithm has been compared with other methods, such as the texture-based LBP and G-LBP. The first sample of each palmprint was considered as the Template, while the other samples were treated as Probes in the experiment. Therefore, there were 200 palmprint Templates and 1800 Probes in total that were matched with all the templates to label. Fig. 5 shows the Receiver Operating Characteristic (ROC) curves for the three methods. ROC reflects the relationship of Genuine Acceptance Rate (GAR) and False Acceptance Rate (FAR) against different judging thresholds. The results indicate that the proposed method has a higher recognition rate and a better overall performance compared to existing methods.

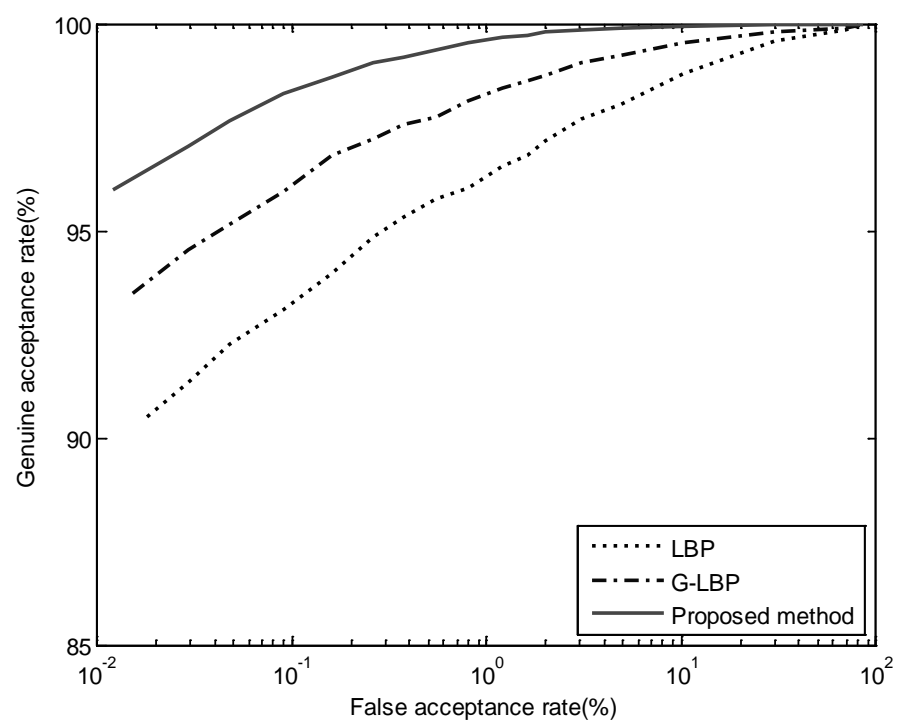

Fig.5 ROC curves with different methods

\section{Conclusion}

This paper presents a palmprint feature extraction method, named G-LDP that combines the Gabor features and LDP characteristics to improve the classification efficiency and overcome the limitations in the existing methods. In the proposed method a multi-directional and multi-scale Gabor filter is used to filter the palmprint images to obtain the global features of palmprint. The Gabor response images are then further divided into sub-blocks and encoded using LDP operator to obtain local palmprint details. Compared to a single feature representation, the proposed method can provide more comprehensive, detailed and accurate information of palmprint features, resulting in improved recognition rate. The experiments on the PolyU palmprint database have proved the effectiveness of the proposed algorithm.

\section{References}

[1] D.D. Zhang, Automated Biometrics: Technologies and Systems., Kluwer Academic, 2000

[2] X. Peng, et al., A Survey of Palmprint Feature Extraction Algorithms, Proc. Intelligent Systems Design and Engineering Applications, 2013 Fourth International Conference on, 2013, pp. 57-63

[3] Yue feng, Zuo wangmeng Zhang dapeng. Survey of Palmprint Recognition Algorithms, AAS, 2010,36(03):353-365(in Chinese)

[4] Shu W, Zhang D. Palmprint verification: an implementation of biometric technology. In: Proceedings of the 14th International Conference on Pattern Recognition. Brisbane, Australia: IEEE, 1998. 219-221 
[5] Zhang D, Shu W. Two novel characteristics in palmprint verification: datum point invariance and line feature matching, Pattern Recognition, 1999, 32(4): 691-702

[6] Pang Y H, Connie T, Jin A T B, Ling D N C. Palmprint authentication with Zernike moment invariants. In: Proceedings of the 3rd IEEE International Symposium on Signal Processing and Information Technology. Darmstadt, Germany: IEEE, 2003. 199-20

[7] Li Y L, Wang K Q, Zhang D. Palmprint recognition based on translation invariant Zernike moments and modular neural network. In: Proceedings of the 2nd International Symposium on Neural Networks. Chongqing, China: Springer, 2005. 177-182

[8] Wu X Q, Zhang D,Wang K Q. Fisherpalms based palmprint recognition. Pattern Recognition Letters, 2003, 24(15): 2829-2838

[9] Du F, Yu P, Li H, et al. Palmprint recognition using Gabor feature-based bidirectional 2DLDA, Computer Science for Environmental Engineering and Eco Informatics, 2011: 230-235

[10]Kong A W K, Zhang D. Competitive coding scheme for palmprint verification. In: Proceedings of the 17th International Conference on Pattern Recognition. Washington D. C., USA: IEEE, 2004. 520-523

[11]Mu, M., Ruan, Q., and Guo, S.: Shift and gray scale invariant features for palmprint identification using complex directional wavelet and local binary pattern, NEUROCOMPUTING, 2011, 74, (17), pp. 3351-3360

[12]F. Shojaiee and F. Hajati, Local composition derivative pattern for palmprint recognition, Proc. Electrical Engineering (ICEE), 2014 22nd Iranian Conference on, 2014, pp. 965-970

[13]T. Jabid, M. H. Kabir, and O. Chae, Local directional pattern(LDP) for face recognition, in Proceedings of the IEEE International Conference on Consumer Electronics, 2010, pp.329-330

[14]Liu C, Weehsler H. Independent component analysis of Gabor features for face recognition. IEEE Transactions on Neural Networks, 2003, 14(4):919-928

[15] Shih F, Chuang C. Automatic extraction of head and face boundaries and facial features[J]. Information Sciences, 2004, 158:117-130

[16] J. G. Daugman, Two-dimensional spectral analysis of cortical receptive field profiles, Vis. Res., vol. 20, no. 10, pp. 847-856, 1980

[17]Zhang D, Kong W K, You J, Wong M. Online Palmprint Identification. IEEE Transactions on Pattern Analysis and Machine Intelligence, 2003, 25(9): 1041-1050 